\title{
Accurate Fingerprint Enhancement and Identification Using Minutiae Extraction
}

\author{
Kumar Attangudi Perichiappan Perichappan, Sreenivas Sasubilli \\ Regional Development Center, KPMG, Roseland, NJ, USA \\ Email:kumarattangudiperich@kpmg.com, ssasubilli@kpmg.com
}

How to cite this paper: Perichappan, K.A.P. and Sasubilli, S. (2017) Accurate Fingerprint Enhancement and Identification Using Minutiae Extraction. Journal of Computer and Communications, 5, 28-38. https://doi.org/10.4236/jcc.2017.514003

Received: November 1, 2017

Accepted: December 11, 2017

Published: December 14, 2017

Copyright $\odot 2017$ by authors and Scientific Research Publishing Inc. This work is licensed under the Creative Commons Attribution International License (CC BY 4.0).

http://creativecommons.org/licenses/by/4.0/

(c) (i) Open Access

\begin{abstract}
Fingerprints are an extraordinary source for recognizable proof of people. Unique finger impression acknowledgment is one of the most seasoned types of biometric identification. However, getting a decent unique finger impression picture isn't that simple. So we must process unique finger impression picture before coordinating. A crucial advance in measurements of fingerprint minutiae is to obtain minutiae from the finger impression pictures dependably. However, fingerprint images are occasionally of perfect quality. They might be debased and defiled because of varieties in skin and impression conditions. Along these lines, image enhancement strategies utilize other details extraction to acquire a more reliable estimation of minutiae areas. The primary objective of this research work is to introduce a superior and improved unique fingerprint image. We studied the elements identifying with getting elite component focuses detection algorithm, for example, picture quality, segmentation, picture upgrade and highlight recognition. Usually utilized features for enhancing unique finger impression picture quality are Fourier spectrum energy, Sobel filter energy, and local orientation. Precise segmentation of unique finger impression edges from a broad foundation is vital. For productive improvement and feature extraction algorithms, we zero the commotion in segmented features. As a pre-processing method, we need to perform comprising of field introduction, ridge frequency estimation, Sobel filtering, division. Then connect the resulting picture to a thinning algorithm and consequent minutiae extraction. After resultant extraction of these minutiae focuses, we will utilize the picture with focuses for coordinating or finding the offenders and also for other security issues. The procedure of image pre-processing and minutiae extraction is explored. The simulations are performed in the MATLAB environment to assess the execution of the implemented algorithm.
\end{abstract}

\section{Keywords}

AFAS (Automatic Fingerprint Authentication System), Fingerprint Image 
Pre-Processing, Minutiae Extraction, Fingerprint Ridge Thinning, Minutiae

\section{Introduction}

\subsection{What Is a Fingerprint?}

A fingerprint is an impression or mark made by a person's fingertip on a surface. It is familiar that, each finger impression is distinct. Everyone's fingerprint is of perpetual uniqueness as shown in Figure 1. A unique mark of an individual is changeless and continues as before, all through their life. We can use the fingerprint to identify the individual from the distinctive pattern of whorls and lines.

A finger print consists of many ridges and furrows on the surface of a finger. Ridges are the upper skin layer sections of the finger and furrows (valleys) are the lower portions. The ridges frame purported minutiae focuses: a ridge finishing, which is known as termination (where a ridge end) and ridge bifurcations (where a ridge parts in two).

We can control the uniqueness of a unique finger impression by the example of ridges, furrows, and the minutiae points. There are five fundamental unique mark designs: arch, tented arch, left side loop, right side loop, and whorl. Circles account for $60 \%$ of all fingerprints, whorls represent $30 \%$, and arches for $10 \%$.

\subsection{What Is a Fingerprint Recognition?}

Fingerprint recognition or fingerprint authentication is the digital technique for checking a match between two human fingerprints performed by AFRS (Automatic Fingerprint Recognition System), which is a program-based. This process is not the same as the manual approach for fingerprint recognition, which the specialists usually follow [1]. The steps for fingerprint recognition are illustrated in the Figure 2.

The fingerprint recognition issue of two sub-domains: one is unique fingerprint verification, and the other is finger impression distinguishing proof.
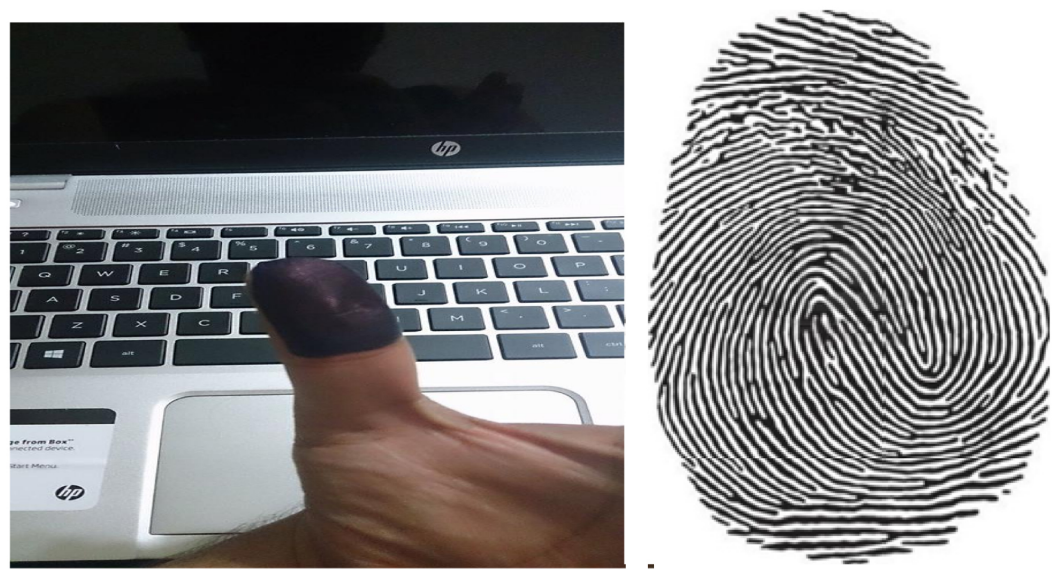

Figure 1. Fingerprint. 


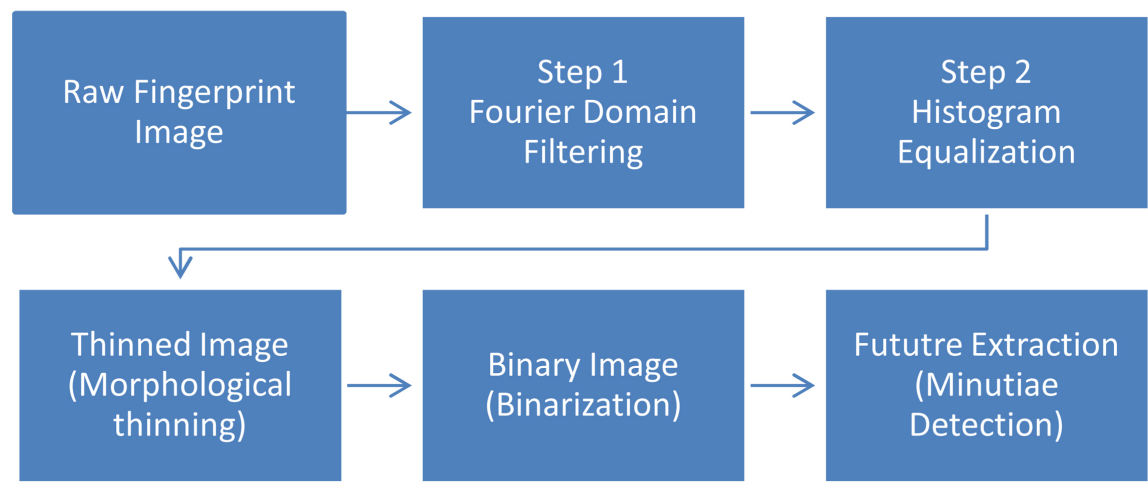

Figure 2. Block diagram for fingerprint recognition.

Fingerprint verification verifies if two fingerprints are from a similar finger or not. The client needs to give their finger stamp together their identity information as for their ID number, and so forth. The fingerprint verification framework recovers the unique mark layout as indicated by the ID number and matches the format with the continuous obtained unique mark from the client. Normally, it is the basic outline rule of AFAS (Automatic Fingerprint Authentication System).

Fingerprint Identification is a technique for ID utilizing the impressions made incrementally ridge designs found on the fingertips. It scans for a query fingerprint in the database.

No two people have the very same course of the arrangement of ridge designs, and the examples stay unaltered for the duration of the life. Other individual attributes may change, yet fingerprints don't. Without the information of a person's identity, the unique mark ID framework tries to coordinate the client's fingerprint(s) with those in the entire unique finger impression database of FVC2002 (Fingerprint Verification Competition 2002). It is particularly helpful for criminal examination of the case that is the principle of AFIS (Automatic Fingerprint Identification System).

However, all the unique fingerprint recognition issues which are either verifying or distinguishing proof based on the characterized portrayal of a finger impression. For whatever length of time that the portrayal of fingerprints remains uniqueness and keeps basic, the finger impression coordinating, either for the 1-to-1 check case or 1-to-m distinguishing proof case, is direct and simple.

\section{System Design}

\subsection{System Level Design}

A fingerprint recognition system consists of a three step process as shown in Figure 3, namely the fingerprint acquiring device, minutiae extractor, and minutiae matcher [2].

For obtaining the finger impression, we utilize an optical or semi-direct sensor which has high proficiency and adequate precision except for a few cases that the client's finger is excessively messy or dry. The database of FVC2002 is used in 
such cases. So here procurement arrange isn't actualized. The minutiae extractor and minutiae matcher modules are further explored in the algorithm part.

\subsection{Algorithm}

To execute a minutiae extractor, we utilize a three-arrange approach given by the analysts, which is illustrated in Figure 4.

For the pre-processing phase of fingerprint picture, Histogram Equalization and Fourier Transform were utilized to make picture improvement. Finger impression picture is then binarized utilizing the locally versatile threshold strategy. The image segmentation assignment is satisfied by a two-step approach: block direction estimation and Region of Interest extraction by Morphological operations (Open and Close). Most of the strategies that we utilize as a part of the pre-processing stage are from different analysts. However, this process shapes a shiny new mix through experimentation [3].

For minutiae extraction arrange, we will go with three thinning algorithms, and the Morphological thinning operation is at last offered out with high proficiency and great thinning quality. The minutiae checking is a straightforward undertaking as most written works detailed. For the post-preparing stage, a more thorough calculation is produced to evacuate false minutiae. Likewise, a new portrayal for bifurcations is proposed to bring together terminations and bifurcation.

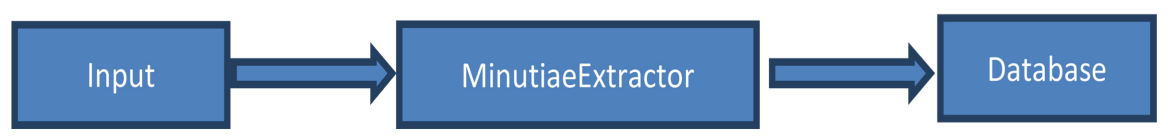

Figure 3. Simplified fingerprint system.

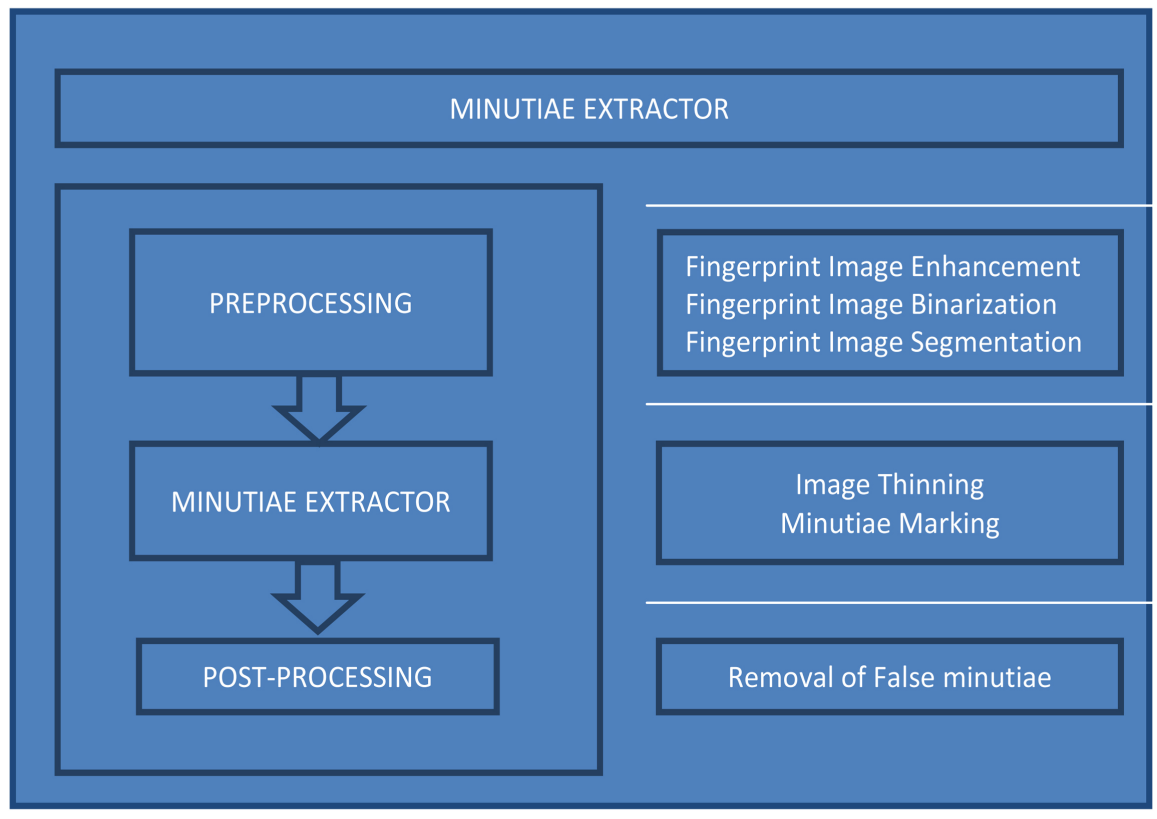

Figure 4. Minutiae extractor. 


\section{Fingerprint Image Processing}

\subsection{Fingerprint Image Enhancement and Acronyms}

Fingerprint Image improvement is to make the picture clearer for further operations. Since the fingerprint pictures procured from sensors with less guarantee with consummate quality, those improvement strategies, for expanding the complexity amongst ridges and furrows and for interfacing the broken false purposes of ridges. Because of the lacking measure of ink, are extremely helpful for keeping a higher precision to fingerprint recognition.

Two Methods, used in the unique mark acknowledgment framework: the Histogram Equalization and the Fourier Transform.

\subsubsection{Histogram Equalization}

Histogram demonstrates the recurrence of the pixels, which are not equivalent. Balance influences the pixels to measure up to, and subsequently histogram balance is utilized to extend the pixel esteem dispersion of the picture to expand the perception data. The first histogram of a unique finger impression picture has the bimodal sort, and the histogram after the histogram adjustment involves all the range from 0 to 255 [4]. The image of Histogram Equalization with Image Enhancement is shown in Figure 5.

\subsubsection{Fingerprint Enhancement by Fourier Transform}

We separate the picture into small blocks ( 32 by 32 pixels) and perform the Fourier transform as given below:

$$
F(u, v)=\sum_{x=0}^{M-1} \sum_{y=0}^{N-1} f(x, y) X \exp \left\{-j 2 \pi X\left(\frac{u x}{M}+\frac{v y}{N}\right)\right\}
$$

For, $u=0,1,2, \cdots, 31$ and $v=0,1,2, \cdots, 31$.

To improve a block by its dominant frequencies, the FFT of the block is multiplies by its magnitude a set number of times.

Where the magnitude of the original FFT $=\operatorname{abs}(F(u, v))=|F(u, v)|$.

$$
g(x, y)=F^{-1}\left\{F(u, v) X|F(u, v)|^{k}\right\}
$$
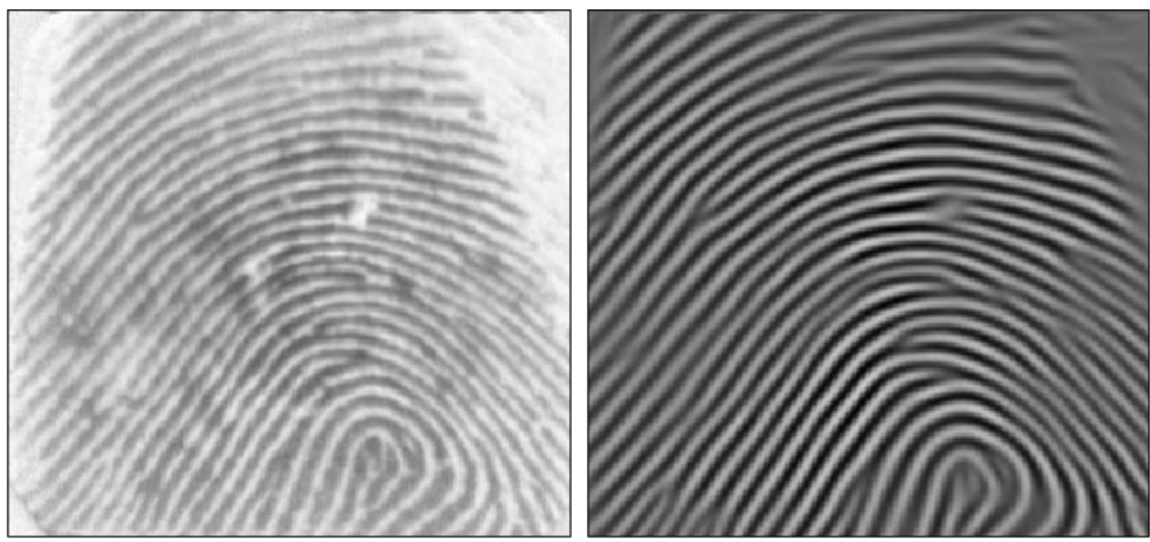

Figure 5. Histogram equalization with image enhancement. 
where, $F^{-1}(F(u, v))$ is given by:

$$
f(x, y)=\frac{1}{M N} \sum_{x=0}^{M-1} \sum_{y=0}^{N-1} F(u, v) X \exp \left\{j 2 \pi X\left(\frac{u x}{M}+\frac{v y}{N}\right)\right\}
$$

For, $x=0,1,2, \cdots, 31$ and $y=0,1,2, \cdots, 31$.

Here the " $k$ " in Equation (2) is a tentatively decided constant, where $k=0.45$ keeping in mind the end goal to figure. While having a higher " $k$ " enhances the presence of the ridges, filling up little gaps in ridges, having too high a " $k$ " can bring about the false joining of edges. Subsequently, a termination may turn into a bifurcation [5].

Upgraded picture is shown in Figure 6, after FFT has the changes to interface some dishonestly broken indicates on ridges and evacuate some spurious (false) associations between the ridges. The reaction of each block is evident however it has no damage to the further operations since the Enhanced Fingerprint was discovered after sequential binarization operation is great if the symptom isn't excessively serious.

\subsection{Fingerprint Binarization Image}

Binarization is a procedure by which the grayscale image is changed over into a black and white image. It is changed over from grayscale to a 1-bit image with 0 -value for ridges and 1-value for furrows. After the operation, ridges in the fingerprint are featured with black color while furrows are white. In this work, threshold (for each $16 \times 16$ ) technique was utilized for Adaptive binarization as depicted in Figure 7 [6].

\subsection{Fingerprint Segmentation Image}

The Segmentation of a fingerprint image area is by contrasting the mean of each $9 \times 9$ block with a threshold value. The mean estimation of the background is higher than the unique finger impression area. Here the threshold is picked by experimentation. We will place the unique segmented fingerprint on a white background.
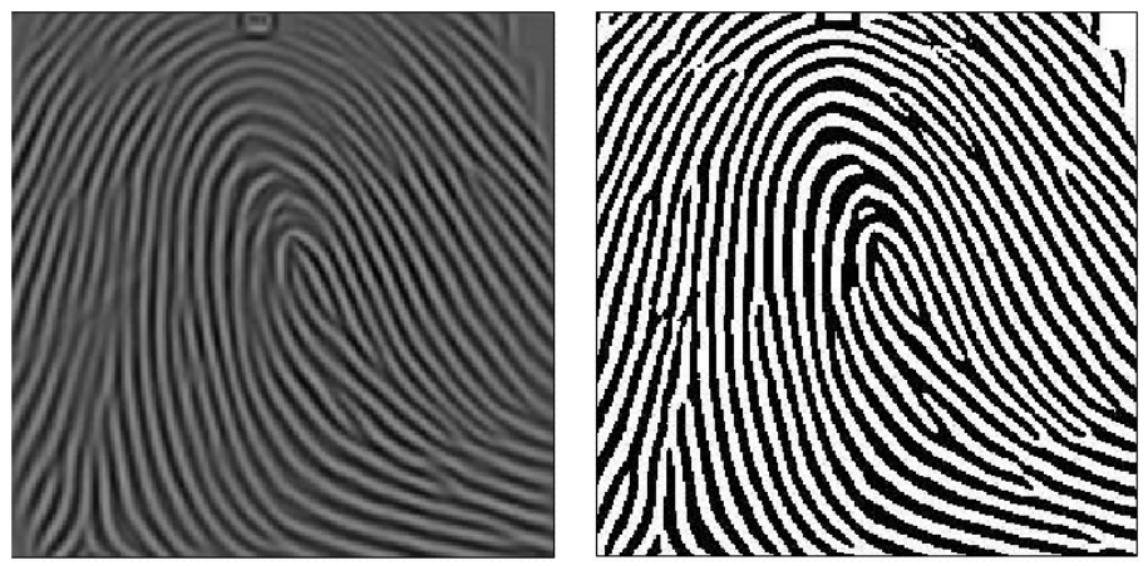

Figure 6. Fingerprint enhancement by FFT. 


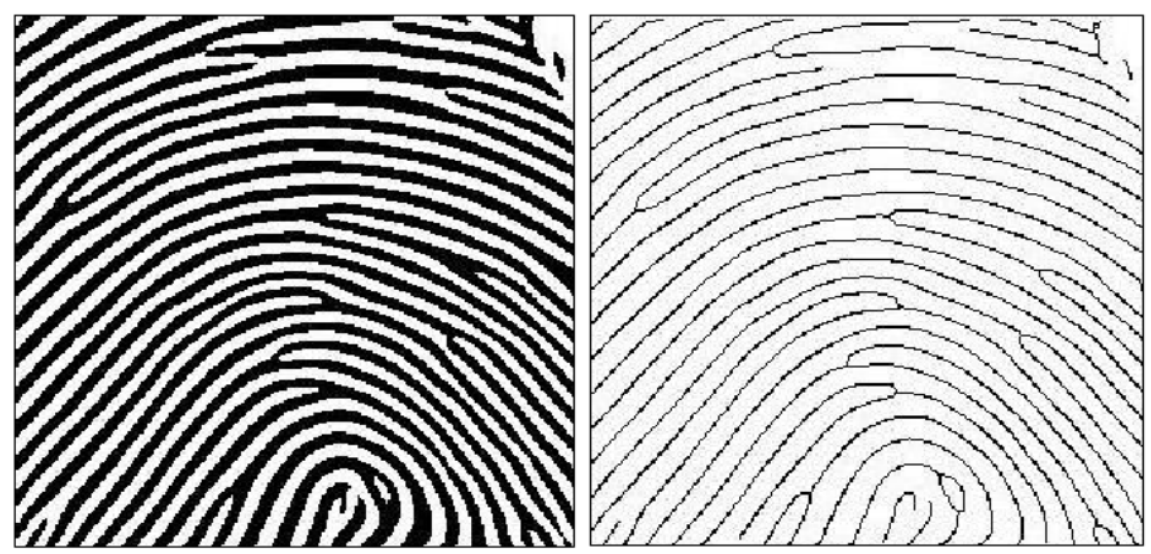

Figure 7. Fingerprint image after adaptive binarization.

Only a Region of Interest (ROI) is helpful to be perceived for each fingerprint picture. The picture range without successful ridges and furrows is first disposed of since it just holds background data. At that point, we will outline the bound of the staying viable range since the minutiae in the binding area is mistaking for those spurious minutiae's, where the ridges are out of the sensor.

To extract the ROI, a two-step technique is utilized. The initial step is block course estimation and the second from some Morphological techniques.

\subsubsection{Block Heading Estimation}

Gauge the blocking heading for each block of the unique finger impression image with $W \times W$ in measure ( $W$ is 16 pixels default). The calculation is:

We compute the slope esteems along $\mathrm{x}$-heading, which is level inclination (gx) and y-bearing, which is the vertical angle (gy) for every pixel of the piece. Hence here we utilize two "Sobel" channels keeping in mind the end goal to satisfy this assignment.

$$
\text { Sobel }=\left[\begin{array}{ccc}
1 & 2 & 1 \\
0 & 0 & 0 \\
-1 & -2 & -1
\end{array}\right]
$$

For each block, utilize the following formula to get the Least Square approximation of the block direction.

If we assume that the vertical slope is cosine and the flat inclination as sine. The introduction of each $16 \times 16$ block of the picture is ascertained utilizing the formula,

$$
\begin{gathered}
\theta_{d}=\frac{1}{2} \tan ^{-1}\left(\frac{\sum_{i=1}^{16} \sum_{j=1}^{16} 2 G_{X}(i, j) G_{y}(i, j)}{\sum_{i=1}^{16} \sum_{j=1}^{16}\left(G_{x}(i, j)^{2}-G_{y}(i, j)^{2}\right)}\right) \\
G_{x} \neq 0 \text { and } G_{y} \neq 0
\end{gathered}
$$

And is simplified as

$$
\theta=\frac{1}{2} \tan ^{-1} \frac{\sum \sum 2 \cos \theta \sin \theta}{\sum \sum\left(\cos ^{2} \theta-\sin ^{2} \theta\right)}
$$




$$
E=\frac{2 \sum \sum(\sin \theta \cos \theta)+\sum \sum(\cos 2 \theta-\sin 2 \theta)}{W * W * \sum \sum(\cos 2 \theta+\sin 2 \theta)}
$$

For each block, if its certainty level $\mathrm{E}$ is below a threshold, then the block is regarded as a background block.

We assume there is only one fingerprint in each image.

\subsubsection{ROI Extraction by Morphological Operations}

Two Morphological operations called "OPEN" and "CLOSE" are used in the ROI extraction. The "OPEN" operation can extend pictures and evacuate crests presented by background noise. The "CLOSE" operation can compress pictures and decrease little depressions.

The subtraction of the closed area from the opened area is called "bound". Then the algorithm throws away those leftmost, rightmost, uppermost and bottommost blocks out of the bound to get the tightly bounded region just containing the bound and inner area also referred to as ROI shown in Figure 8 [7] [8].

\section{Accurate Fingerprint Enhancement and Identification Using Minutiae Extraction}

\section{Fingerprint Ridge Thinning}

Thinning is an operation by which decreases the widths of the ridges. An inbuilt function called BWMORPH will be useful for this.

In each scan of the full finger impression picture, the algorithm marks the excess pixels in every single picture window $(3 \times 3)$ as illustrated in Figure 9 . Lastly, deletes each one of those stamped pixels after a few scans. The thinning edge outline at that point separated by other three Morphological operations to expel some $\mathrm{H}$ breaks, secluded focuses and spikes [1].

This sample along with other information will be logged into the database. Consistently detail of the unique finger impression will be gotten with no uncertainty. This procedure is likewise helpful for the scientific individuals to get the more details of the offender [6].
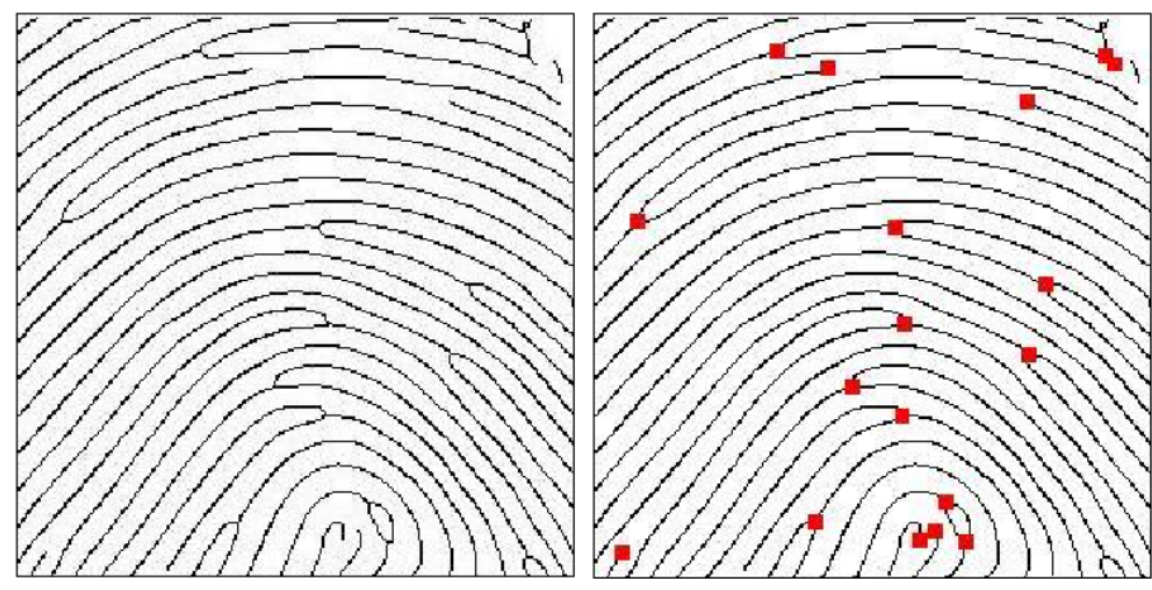

Figure 8. Region of interest (ROI). 


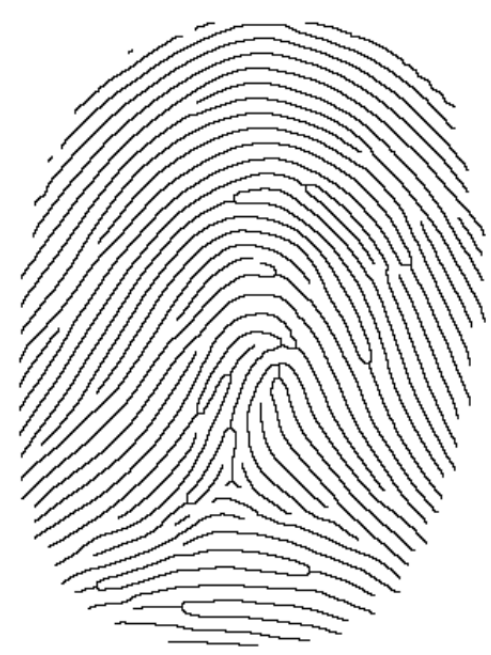

Figure 9. Fingerprint ridge thinning.

We composed the code in MATLAB to execute this. The entire procedure needs a portable workstation with MATLAB programming and a camera versatile to catch the impressions of the fingers. The procedure of execution is given as under:

- Initially, an impression will be considered and that will be stacked into the framework by the assistance of a mobile camera.

- This alleged record will be given as input file. The condition for that record is to be in .tif design (in this research).

- After getting this input file, the handling will be done over it to get each moment points of interest.

- Depending on the nature of the picture, the picture upgrade program will be finished. The pixel clearness will be progressively and that will help in discovering great things. FFT will be utilized for this reason.

- Then it will go under binarization, where at that point lines will be a settled example, say like dark on white or white on dark.

- Later, every single gap will be filled consequently, if the gap is up to significant length. By that, the bearings of the lines will be created. With these, we will get some thought of the picture on which, many the segments were framed by the programmed and slight extensions or projections.

- Now we need to consider the district of intrigue, this will dispense with the undesirable picture divide around the required specimen, and focuses chiefly on the impression territory. i.e., the primary part in the unique mark.

- Finally, it will distribute shading all bifurcation focuses with one color and terminating focuses with a different color. Consistently insight about the unique mark will be acquired.

\section{Conclusions}

The primary objective of this research work is on the upgrade of fingerprint pictures, and the sequential extraction of minutiae. Progression of Methodologies 
for fingerprint picture was implemented to extract the minutiae.

We can classify these methodologies basing on the following components:

1) The sort of info pictures they handle, i.e., regardless of whether binary or grayscale.

2) Strategies of binarization and segmentation included, whether the thinning is required or not.

3) The necessary measure of exertion in the post handling stage if exists.

In any case, low-quality fingerprint pictures need pre-processing to expand differentiation, and lessen various sorts of noise as loud pixels additionally create a lot of spurious minutiae they get upgraded efficiently between pre-processing steps.

Initiation of each method might be one of these pre-processing steps, but the final output will be the same minutiae, which will be the key to the further investigation.

\section{Future Scope}

The future extent of the work is to enhance the quality of the fingerprint image either by improving the equipment to capture the picture or by using the next level picture upgrade techniques so that we can improve the input image for the thinning stage. This would in turn enhance the results from the three arrange approach process.

In most cases, we may not get the complete picture of the finger, if we include the concepts of image analysis with this, we can build rest of the image from the available portion. We have identified the possible future scopes where this can be applied to as illustrated below.

The enhanced thinning concept will play a key role. Top two main features of the idea are:

1) We can diminish the picture to single pixel width which will help us from a storage point of view. As we can rebuild the entire image based on the individual pixels, we can get back the original image from these building blocks.

2) Some bifurcations can be distinguished, which were missed before because of the nearness of pixels in the diminished picture. This property will provide complete detail of the print, which will reduce the time to match it with the file from the database.

The picture with light print or super low pixel and contorted pictures additionally ought to be prepared for the improvement in the adjoining or anticipating part. Enhanced thinning will help here in removing the outer part of interest area and outlining an artificial neural system classifier for fingerprints given minutiae highlights.

Forensic Department can use this advanced version to match the available prints with the records of accused candidates. Just provide any finger impression on the weapon, it will give the details of the candidate who used it. We can also train to analyze the impression on the body or any uneven surfaces too. 


\section{References}

[1] Maio, D. and Maltoni, D. (1997) Direct Gray-Scale Minutiae Detection in Fingerprints. IEEE Transactions on Pattern Analysis and Machine Intelligence, 19, 27-40. https://doi.org/10.1109/34.566808

[2] Igaki, S., Shinzaki, T., Yamagishi, F., Ikeda, H. and Yahagi, H. (1992) Minutia Data Extraction in Fingerprint Identification. US Patent No. US5109428 A.

[3] Afsar, F.A., Arif, M. and Hussain, M. (2004) Fingerprint Identification and Verification System Using Minutiae Matching, PIEAS.

[4] Jomaa, D., Dougherty, M. and Fleyeh, H. (2010) Segmentation of Low Quality Fingerprint Images. 2010 International Conference on Multimedia Computing and Information Technology (MCIT), Sharjah, 2-4 March 2010.

http://ieeexplore.ieee.org/abstract/document/5444844/

[5] Iwai, R. and Yoshimura, H. (2011) Matching Accuracy Analysis of Fingerprint Templates Generated by Data Processing Method Using the Fractional Fourier Transform. International Journal of Communications, Network and System Sciences, 4, 24-32. http://file.scirp.org/Html/3-9701272_3692.htm

[6] Jain, L.C., Halici, U., Hayashi, I., Lee, S.B. and Tsutsui, S. (1999) Intelligent Biometric Techniques in Fingerprint and Face Recognition. The CRC Press, Boca Raton, FL.

[7] Ratha N., Chen, S. and Jain, A.K. (1995) Adaptive Flow Orientation Based Feature Extraction in Fingerprint Images. Pattern Recognition, 28, 1657-1672. https://doi.org/10.1016/0031-3203(95)00039-3

[8] Lin, H. (1998) Automatic Personal Identification Using Fingerprints. Ph.D. Thesis, Michigan State University, East Lansing, MI. 